\title{
Identificando a trabajadores legales mexicanos en la frontera de Estados Unidos: percepciones y decepciones en el análisis de la migración fronteriza
}

\author{
Ellwyn R. Stoddard \\ Universidad de Texas en El Paso
}

\begin{abstract}
Resumen
Este artículo profundiza en dos grupos: el de los inmigrantes mexicanos legales y los trabajadores ilegales, quienes se encuentran tan estrechamente integrados dentro de la economía fronteriza de Estados Unidos, que cualquier intento hecho por investigar minuciosamente la condición legal de la población hispanoparlante en el suroeste, a fin de determinar algún tecnicismo legal para identificarlos, tendría devastadoras consecuencias para el suroeste, mismas que serían sentidas en toda la economía norteamericana. Si las políticas de inmigración para el futuro pretenden dar solución al problema de la migración y control sobre la frontera, deben primero entender las realidades funcionales de ésta y formular una política binacional basada en la existencia de los patrones de control informal.
\end{abstract}

Palabras clave: inmigrantes legales, trabajadores ilegales, migración, políticas inmigratorias, economía fronteriza.

\begin{abstract}
This article researches on two groups: the group of legal Mexican immigrants, and the group of illegal workers, who are closely integrated into the border economy of the United States. If we try to thoroughly start a research on the legal status of the Hispanic speaking people of the Southwest of the United States, in order to detect some legal technicality to identify them, it would have devastating consequences to the Southwest that would have effects on the whole American economy. If future immigration politics pretend to solve the migration problem and to control the border, these should first understand its functional realities and establish a bi-national politics that has patterns of informal control as a basis.
\end{abstract}

Keywords: legal immigrants, illegal workers, migration, immigration politics, border economy. 


\title{
IDENTIFICANDO A TRABAJADORES LEGALES MEXICANOS EN LA FRONTERA DE ESTADOS UNIDOS; PERCEPCIONES Y DECEPCIONES EN EL ANALISIS DE LA MIGRACION FRONTERIZA *
}

\author{
Por \\ Ellwyn R. Stoddard **
}

Con el propósito de poder discutir razonablemente los impactos y consecuencias que la fuerza de trabajo legal mexicana ejerce en la frontera de Estados Unidos, estos trabajadores deben ser identificados y diferenciados de los trabajadores ilegales mexicanos en esa misma región.

Abundan las concepciones ficticias acerca de estas dos categorías que componen el mercado laboral extranjero del suroeste, considerándolas como entidades separadas y diferenciables entre sí. Nada puede estar más alejado de la verdad. En realidad, las nociones de trabajador legal e ilegal, sólo constituyen los polos de un continuum en el cual la mayoría de los trabajadores resultan ser más "legales" que otros; mientras que otros que laboran en el mismo campo o centro de trabajo, son más "ilegales" que otros. Las políticas de inmigración vagas y vacilantes, los procesos de reforzamiento de control, los parches a las leyes, el mismo desarrollo histórico y la continuación de redes fronterizas informales, en conjunto, confunden el tema de quién permanece legal y quién ilegal, a tal grado de que la confianza en tal determinación, seguido se deja a percepciones normativas caprichosas que resultan inconsistentes con los criterios legales. Más aún, los conceptos de legalidad e ilegalidad, como comúnmente se usan en los Estados Unidos, en su aplicación se inclinan hacia el concepto de "ilegal", de manera similar a la pregunta académica falsoverdadero. Si una pregunta es completamente verdadera en todos sentidos, es verdadera. Si solamente una parte es falsa, la pregunta entera debe ser considerada falsa. $O$ puesto de otra manera, la frase "dólares y centavos" (pesos y centavos), parece denotar igualdad en cualquier designación de "pennies"*** hasta llegar a la denominación más grande, que es el dólar. Sin embargo, cualquier cantidad de uno a 99 centavos se considera como "centavos"; mientras que sólo la suma de 100 pennies, o de 100 designaciones, recibe propiamente la designación de "dólar". De este modo, los polos de "trabajador legal" y "trabajador ilegal" son regular-

\footnotetext{
- Traducido por Oscar García Sáenz y Guadalupe Ortega Villa.

Documento presentado en la "Conferencia invitacional sobre fuerza de trabajo legal mexicana en la frontera de Estados Unidos". Llevada a cabo en la Universidad Autónoma de Baja California, Mexicali, B.C., el 29 de octubre de 1984. Mi especial reconocimiento al Sr. Al Velarde, Director de la Conferencia Católica Estadounidense para los Servicios Migratorios a los Refugiados, por su ayuda con la Ley de Inmigración y sus requisitos.

* Profesor de sociología y antropología de la Universidad de Texas en EI Paso.

** En Estados Unidos la palabra "pennies" se utiliza para designar centavos.
} 
mente claros y reconocibles. Pero la mayor parte de las denominaciones entre estos polos son mezcla de elementos de legalidad e ilegalidad. Por tanto, el debate de la fuerza de trabajo legal mexicana debe incluir el debate de la fuerza de trabajo ilegal mexicana, aunque sea como un medio para determinr el significado de la fuerza de trabajo de la cual estamos hablando.

Después de un examen rápido de nuestra historia de la inmigración legal de mexicanos a los Estados Unidos, algunas leyes actuales y procedimientos utilizados para determinar la legalidad o ilegalidad de los extranjeros mexicanos, serán examinados para demostrar su falta de consistencia. Finalmente, será analizado el papel de las redes informales fronterizas como un regulador cuasi oficial de los trabajadores mexicanos, para demostrar que periódicamente hasta las politicas gubernamentales estadounidenses violan sus propios mandatos legales en busca de la estabilidad económica dentro de sus propias fronteras nacionales.

\section{UN SIGLO DE POLITICA INMIGRATORIA Y NO-POLITICA HACIA LOS MEXICANOS}

Hasta la segunda década del siglo XX, las restricciones para inmigrantes de México fueron tan limitadas, que existieron pocas razones para que las personas que entraron de ese país lo hicieran como ilegales. Cuando el Acta de Inmigración de 1917 se inició, ésta estableció un impuesto de ocho dólares por cabeza para los inmigrantes mexicanos, acompañado de una difícil "prueba de escritura y conversación"; en ese entonces la inmigración sólo fue escasamente impedida a aquéllos que buscaban la entrada legal. En 1924, con 500 hombres se creó la Patrulla Fronteriza como parte del Servicio de Inmigración y Naturalización (SIN), iniciándose así los intentos reales para controlar la migración mexicana. Anteriormente, un puñado de guardias texanos (Texas Rangers), grandes en tradición y pequeños en sensibilidad étnica, caprichosamente regularon el flujo migratorio México-Estados Unidos. Después de la creación de la Patrulla Fronteriza, a ésta se le asignó la función de control.

El Acta de Inmigración de 1924 restringió la inmigración hacia Estados Unidos procedente de las regiones "indeseables", a fin de que la composición étnica de los Estados Unidos coincidiera con la de 1890. Aunque México nunca fue incluido en las restricciones numéricas por esa acta o por ninguna de las medidas anteriores que trajeron como consecuencia la exclusión de los orientales y el establecimiento de los criterios restrictivos de inmigración, a medida que se incrementó el número de mexicanos y de sus descendientes, su presencia se hizo cada vez más notoria como una responsabilidad económica, aunque su estancia en el suroeste fue para proporcionar el trabajo necesario para continuar con 
el orden económico de esa región. ${ }^{1}$ Evidentemente, las regulaciones morales y legales estuvieron en desacuerdo con las necesidades reales del mercado laboral del suroeste, el cual continuaba con sus modelos extra legales de reclutamiento y uso de trabajadores mexicanos, con la bendición informal del gobierno de Estados Unidos.

Durante los años de la Segunda Guera Mundial, Estados Unidos se acababa de recuperar de la década de la Gran Depresión mundial. La inmigración mexicana legal e ilegal fue promovida entonces para cumplir con las necesidades laborales de los Estados Unidos en los sectores de agricultura y servicios.

En forma bilateral se formalizó un programa "bracero", establecido entre los gobiernos federales de México y Estados Unidos, mediante el cual, trabajadores de las áreas mexicanas de alto desempleo y pobreza fueron trasladados masivamente hacia los Estados Unidos durante las temporadas críticas de cosecha.

A diferencia del primer programa unilateral de braceros iniciado por Estados Unidos después de la Primera Guerra Mundial, este convenio contenía garantías explícitas en cuanto a condiciones de trabajo, escalas salariales, vivienda, transporte, etc., mismas que fueron supervisadas muy de cerca para mantener el estricto cumplimiento de esas normas.

Este programa continuó formalmente (a veces informalmente) hasta su término en 1964, y constituyó un medio para que los trabajadores mexicanos se familiarizaran con los propietarios de los ranchos, con quienes en el futuro obtendrían empleo como trabajadores ilegales. Dé ese modo, el uso controlado y formalizado de trabajo mexicano resultó ser una "bomba alimentadora" de las subsecuentes olas de trabajadores mexicanos "ilegales", perseguidos, protegidos y utilizados por los intereses norteamericanos.

La nueva Acta de Inmigración de 1965, disminuyó gradualmente las anteriores cuotas de inmigrantes asignadas para cada país que habian sido creadas en 1924; y por primera vez los inmigrantes de México fueron restringidos numéricamente. Se les incluyó dentro de la asignación para el Hemisferio Occidental con 120,000 visas anuales y un límite de 20,000 personas por nación.

Este constreñimiento jurídico puso una barrera a los más de 60,000 mexicanos que anualmente habían entrado a los Estados Unidos a través de los conductos legales; de manera que, a medida que el flujo migratorio continuó, también continuaron los flujos normales de mexicanos buscando un status legal dentro de Estados Unidos; pero dos tercios de ellos se encontraron en la categoría de extranjeros ilegales en lugar de ser incluidos en el proceso legal tradicional como en años anteriores. Así, un

1 García y Griego, Manuel. Mexico and the United States: Migration, History and the Idea of Sovereighty. EI Paso: University of Texas at EI Paso, Center for Inter-American and Border Studies, Border Perspectives No 7. December. 1983. p. 13. 
caprichoso decreto sobre inmigración intentó restringir la migración mexicana, logrando únicamente el cambio en la clasificación de dos tercios de aquéllos que entraban en forma "Iegal", pasando ahora a ser "ilegales". Evidentemente, los trabajadores mexicanos que fueron atrapados en tales maquinaciones en el transcurso de un año pasaron a ser clasificados como trabajadores ilegales, por hacer lo que ellos mismo hicieron legalmente durante los 10 años anteriores.

Entretanto, en los campos, aquel mismo trabajador pizcó algodón o remolacha; recolectó papas, manzanas y cerezas en esos mismos huertos, donde ahora algunos eran ilegales y el resto permanecía legal.

De ahora, 1968 a 1977 , aproximadamente el $35 \%$ de todos los nuevos inmigrantes a Estados Unidos eran hispano-parlantes; sin embargo, México no continuó siendo el más grande abastecedor de inmigrantes legales, ya que algunos países latinoamericanos utilizaron su cuota de visas hasta un máximo de 20,000 por año. ${ }^{2}$

En los consulados estadounidenses en México, rápidamente se incrementó la lista de solicitudes para residir en Estados Unidos por un período de cinco a diez años, mismas que siguen creciendo a pesar de las reformas legales que permiten un poco más de inmigración proveniente de Canadá y México, con fundamento en la "prioridad de los vecinos". Aun cuando las tres décadas de activismo en derechos civiles, aunadas a la creciente fuerza de los votantes de habla hispana en el suroeste, han estimulado una actitud nacional más tolerante hacia la gente de "piel morena" (acerca de las cuales una gran parte del público norteamericano supone que son extranjeros mexicanos), parece que cualquier intento para modificar las actuales politicas de inmigración con legislaciones similares a la iniciativa de Ley Simpson-Mazzoli ${ }^{3}$ - la cual feneció recientemente en el comité de la Conferencia de la Casa de Representantes del Senado de Estados Unidos-, tendrá que vencer la inercia de las doctrinas atrincheradas a las cuales se les confiere respeto verbal, pero que continuamente son quebrantadas por las demandas, tanto del mercado laboral del suroeste, como por el suministro inadecuado de trabajadores para ocupaciones domésticas.

\section{CRITERIOS EMPLEADOS PARA DETERMINAR LA CONDICION LEGAL DE EXTRANJEROS MEXICANOS APREHENDIDOS.}

La aprehensión de inmigrantes ilegales mexicanos se lleva a cabo usualmente cuando las personas cruzan hacia los Estados Unidos en busca de empleo, o bien en sus lugares de trabajo o en tránsito hacia o desde el

2 Teiltelbaun, M. "Right versus Right: Immigration and Refugee Policy in the United States". Foreign Affairs. 59 (No 1). 1980. pp. 26-27.

${ }^{3}$ Keely, Charles B. "Current Status of U.S. Immigration and Refugee Policy". In: Mary M. Kritz (ed) U.S. Immigration and Refugee Policy. Lexington: D.C. Heath (Lexington Books). 1983. 
centro de trabajo remunerado. Rara vez, si es que nunca, se allana un domicilio, forzando a las mujeres y niños que se encuentran dentro a identificarse y a dar pruebas de su permanencia legal. Por eso, cuando hablamos de inmigración legal mexicana, en realidad estamos hablando de trabajadores mexicanos presentes o potenciales, indiferentemente de su condición legal.

Cuando una persona es aprehendida y llevada ante las autoridades para una audiencia legal, la determinación de cada caso está sujeta a los diferentes decretos y disposiciones que amparan su situación, sea que esté o no consciente de su existencia. Pero estas disposiciones tratan diferencialmente, tanto casos de residencia de largo o corto plazo, como actividades de trabajo o de no trabajo. Mientras que en algunos casos, contar con un empleo fijo y lucrativo puede ser motivo para que proceda una petición de residencia permanente; en otros, la existencia de un empleo deniega una solicitud para obtener la condición legal de residente extranjero. De ahí que, además de la aprehensión del trabajador mexicano, quien es formalmente acusado y "juzgado" en la corte de inmigración, muchos de los trabajadores mexicanos que trabajan en los campos del suroeste o en las industrias de servicios urbanos son "ilegales" sin saberlo. Y algunos de aquéllos que se perciben a sí mismos como "ilegales", son trabajadores legales en todo sentido, excepto por el simple hecho de haber demandado que su permanencia se legalice.

Para presentar algunas de las interpretaciones mutuamente contradictorias que comúnmente se utilizan para determinar la legalidad de algunos trabajadores mexicanos aprehendidos en los Estados Unidos, serán examinadas algunas situaciones que describen e ilustran esta confusión.

Entrada legal. La entrada ilegal de un ciudadano mexicano puede ocurrir de muchas maneras en diferentes circunstancias legales. Algunos entran sin identificación o indocumentados. ${ }^{4}$ Otros entran con documentos legítimos que no les pertenecen y los cuales son manufacturados o utilizados fraudulentamente. Otros entran con una visa de entrada legítima, pero se convierten en explotadores de ésta cuando sobrepasan los límites de tiempo en ella señalada, o se contratan en actividades que están prohibidas bajo las concesiones de la visa. Esto último incluye el torrente de trabajadores domésticos, jardineros y trabajadores por día, quienes vienen hacia los Estados Unidos con visas de "visitantes". También aquellos trabajadores a quienes se les ha concedido permisos de trabajo otorgándoseles las "tarjetas verdes" para trabajar en empleos considera-

\footnotetext{
4 El eufónico (y falso) uso común del término "indocumentado", como sustituto del término inmigrante ilegal mexicano, es muy engañoso. Los indocumentados son solamente una parte de la migración ilegal, y por eso técnicamente no es un término genérico para definir todo el fenómeno. Por otra parte, sus condiciones legales están determinadas por las leyes y por eso la migración ilegal es un producto de la asignación de una condieión "legal" o "ilegal". De aquí que el término más preciso sea extranjero ilegal mexicano.
} 
dos como no ocupables por ciudadanos estadounidenses, según los estudios del Departamento Federal del Trabajo, pueden entrar a Estados Unidos legalmente; pero al obtener un trabajo fuera del sector definido por los requerimientos laborales que permitieron la concesión de la tarjeta verde, se convierten en empleados ilegales.

Aquellos trabajadores mexicanos tenaces que entran a los Estados Unidos ilegalmente por cualquiera de los medios arriba mencionados, deben continuar trabajando como ilegales, ya que el hecho de haber laborado ilegalmente les impide calificar para obtener su condición de extranjero legal. Según la sección 245 del Código de Inmigración de 1952, sólo aquellas personas que entraron legalmente y que no hayan trabajado en los Estados Unidos, están posibilitados para que proceda la solicitud de cambio de su condición legal. Así, en una nación que se enorgullece del trabajo tenaz de sus primeros inmigrantes europeos, recompensa a aquéllos que entran legalmente sin trabajar mientras que penaliza a los que entran legal o ilegalmente, y procuran su propio sostenimiento a través de un trabajo honrado. Tales procedimientos legales reflejan la manera de pensar torcida de quienes hacen las políticas, así como de los legisladores, en la medida en que se alejan de la realidad funcional dirigiéndose hacia el aire rarificado de la juris ridícula. Cuando las leyes desafían abiertamente los valores fundamentales de una sociedad, éstas son rebasadas por medio de las redes funcionales establecidas para continuar con los modelos tradicionales binacionales de la frontera.

Los puristas se refieren a esto como una "brecha" entre la frontera de Estados Unidos y la soberanía norteamericana, pero el apoyo institucional para modelos tradicionales de uso de trabajo mexicano, continúan tan fuertes ahora como en décadas pasadas. ${ }^{5}$

Residencia ininterrumpida en Estados Unidos. Según la sección 249 del Código de Inmigración de 1952, se puede otorgar ayuda administrativa (que popularmente se conoce como amnistía) a ciertos peticionarios que hayan residido continuamente en los Estados Unidos desde el 24 de junio de 1949. La reciente iniciativa de la ley Simpson-Mazzoli, que no fue aprobada por el Congreso de los Estados Unidos antes de que terminaran las sesiones en el otoño de 1984, contemplaba medidas para actualizar esta situación desde 1972 en adelante, de acuerdo con la exposición de la propuesta de ley que se había discutido. Según la sección 244-A, se podía obtener una condición cuasi legal, en donde la suspensión de las deportaciones permitiría la residencia continua en los Estados Unidos al cumplirse con los tres criterios siguientes: $a$ ) residencia ininterrumpida en los Estados Unidos durante siete años, probándose que la persona no abandonó el país para visitar, atender responsabilidades familiares u otras

\footnotetext{
s Ver: Stoddard, "Ilegal Mexican Labor in ter Borderlands: Institutionalized Support of an Unlawful Practice". Pacific Sociological Review. 19 April, 1973, pp. 175-210.
} 
razones; b) que la persona goce de buena salud y de buenos principios morales (este último punto se refiere a criterios jurídicos); ${ }^{6} c$ ) que la familia o alguno de sus miembros, padezca privaciones u opresiones (causas diferentes que la mera necesidad de buscar la estabilidad económica por medio del empleo en los Estados Unidos), frecuentemente aplicado a personas que buscan asilo de las intimidaciones políticas en sus países de origen.

En algunos ajustes de las clasificaciones legales amparadas por la sección 245 de la Legislación de Inmigración de 1952, algunas apelaciones de la familia se considerarían de peso para que se les concediera una visa permanente o la residencia legal no clasificada en los Estados Unidos; el matrimonio con un ciudadano norteamericano o el ser padre de un niño (nacido de un ciudadano estadounidense o nacido en Estados Unidos) quien a fin de cuentas podría solicitar que se le reuniera en Estados Unidos con el (los) padre (s) extranjero (s), a fin de "restablecer"la unidad familiar. El solicitante debe de haber efectuado su entrada a los Estados Unidos en forma legal, debe tener alguna apelación de tipo familiar (por ejemplo, la necesidad de restablecer la unidad familiar); disponer de una visa (que debido a la gran cantidad de solicitudes pendientes de resolu. ción, significa una espera de muchos años), y no debe de haber obtenido empleo remunerado durante su permanencia en los Estados Unidos. La persona que vive de alguna de las formas de ayuda pública o privada, está legalmente posibilitada para solicitarla; en tanto que la persona que bajo su responsabilidad se incorpora a la fuerza de trabajo y gana dinero para su sostenimiento y el de su familia, no tiene dicha posibilidad. Tal disposición jurídica es un atentado a los valores básicos norteamericanos; recompensa al irresponsable y dependiente mientras que penaliza al trabajador activo, tenaz y autosuficiente. Con el fin de estar en posibilidad de mantener una "tarjeta verde" para algún trabajo aprobado por el Departamento de Trabajo de los Estados Unidos - ya que algunas labores no son ocupadas por ciudadanos estadounidenses- el trabajador debe laborar un mínimo de tres días por semana para asegurar su derecho a seguir haciendo uso de ese documento. Si el trabajador regresa a México para atender a la familia, asistir a funerales, o por alguna situación especial que promueva la cohesión familiar, su falta de trabajo continuo o de residencia, se convierte en un riesgo legal para mantener su permiso de trabajo y su condición "legal" dentro de la fuerza de trabajo de los Estados Unidos. No es de asombrarse por qué la región fronteriza se ha convertido en un sistema informal de inmigración tradicional y de modelos de trabajo que, a pesar de ser técnicamente ilegales cuando se compa-

\footnotetext{
- Si un mexicano es aprehendido por segunda ocasión al tratar de cruzar a los Estados Unidos ilegalmente, incurre en delito. Sin embargo, si reconoce su culpabilidad y acepta la repatriación voluntaria, puede regresar con su familia con base en la suspensión de la sentencia. Pero si posteriormente esta persona trata de solicitar la ciudadanía, se le considera como un "riesgo peligroso", debido a su tendencia delictuosa previa, por las múltiples entradas ilegales no-criminales.
} 
ran con elementos absurdos de las políticas inmigratorias, continúan recompensando la iniciativa personal, las responsabilidades familiares y necesidades simbióticas trabajador-patrón las cuales son de beneficio mutuo en ambos lados de la frontera.

\section{REDES INFORMALES Y TRABAJO ILEGAL MEXICANO EN LA FRONTERA}

La presencia de redes tradicionales informales que unen la frontera político-jurídica entre México y Estados Unidos ha sido descrita y verificada en forma detallada. ${ }^{7}$ Estas redes son cuidadosamente mantenidas por intereses fronterizos, debido principalmente a los mandatos jurídicos caprichosos e irreales que han sido formulados por los hacedores de políticas alejados de la región fronteriza, y desinformados acerca de sus condiciones, necesidades o requisitos mínimos para sobrevivir como cultura. Dichas redes han desarrollado medios para legitimar a los trabajadores mexicanos, quienes técnicamente pudieran ser legales fuera de las interpretaciones estrictas de la ley de inmigración. La idea de que la frontera nacional es sólo un lugar en donde dos sistemas nacionales coinciden, ha sido descartada y reemplazada por la realidad de que ambas culturas, la mexicana y la norteamericana, así como las instituciones, gentes, economías y culturas, rebasan las fronteras nacionales en el limite del estado-nación. Se ha llegado a entender que una frontera tiene más que la mera función de impedir y permitir la salida de gente, bienes y servicios; sino que también opera para inerementar el comercio fronterizo, el turismo y la interacción amistosa, mismas que atraviezan el escenario fronterizo. Por eso, muchos de los trabajadores mexicanos considerados en las estadísticas como "trabajadores legales mexicanos", han sido legitimados por procedimientos de instituciones fronterizas, a pesar de que ellos quizá de hecho sean técnicamente "ilegales" de una u otra forma. Los ciudadanos mexicanos que entran a los Estados Unidos ilegalmente, han sido clasificados con hase en sus motivos de llegada, los cuales a su vez dictan sus patrones de encubrimiento, apoyo económico y/o planes para obtener la eventual ciudadanía estadounidense. Uno de estos dos grupos de ilegalidad surge del proceso formal para la obtención del acceso legal a Fistados Unidos; debido a errores ya sea técnicos, de procedimientos o administrativos por parte de la agencia tramitadora y/o de su clientela, ellos son erróneamente admitidos en los Estados Unidos, aun cuando han violado alguna regla, al firmar un papel en el lugar equivocado, o invirtiendo los datos de fecha/mes de nacimiento, o cualquier otra infracción menor. Estos "ilegales" son posteriormente

\footnotetext{
${ }^{7}$ Stoddard, Ellwyn. Functional Dimensions of Informal Border Networks. El Paso: University of Texas at EI Paso, Center for Inter-American and Border Studies, Border Perspectives Ne 8 (January). 1984.
} 
considerados trabajadores/residentes legales mexicanos, a pesar de que técnicamente han violado los trámites inmigratorios.

El segundo grupo de ilegales, clasificados en función de las principales causas que los orillaron a entrar a Estados Unidos, incluye seis tipos o categorías brevemente resumidas a continuación.

Aventureros: Jóvenes y vándalos fastidiosos, quienes utilizan la frontera como una zona protegida para sus aventuras; ventajosos: concepción errónea de los extranjeros ilegales que viven de la "caridad", versión que es perpetuada por los medios de comunicación pero de la que se ha descubierto su total falsedad, según los resultados obtenidos por investigadores científicos; refugiados por causas económicas: incluye posiblemente el $90 \%$ o más de todos los extranjeros ilegales - quienes comúnmente son los subempleados o desempleados en México y buscan mejores oportunidades de empleo en Estados Unidos-; pre-ciudadano quien usa su tiempo y sus contactos en los Estados Unidos preparándose para solicitar eventualmente su ciudadanía: criminal: ésta es la misma clasificación y se usa en el mismo sentido que si se tratara de un inmigrante legal o un ciudadano nativo; y camaleón: quien entra ilegalmente y cambia tanto su modo de vida, que es aceptado como un ciudadano legal y es capaz de ocultar su origen ilegal, y asi ascender a ocupaciones de tipo profesional y directivas. ${ }^{8}$

La diversidad de estos tipos y la implicación de que algunos "sean legitimados" sin pasar por los medios legales del SIN, sugiere que todos los inmigrantes pueden o no ser identificados fácilmente; $y$ todos aquéllos que pueden ser identificados no encajan completamente en ninguna de las categorías de inmigrante ilegal o inmigrante legal.

Bajo ciertas condiciones, tales como el ser miembro de un sindicato en California, las regulaciones locales o los estatutos locales impiden la revisión de los datos del trabajador para determinar su residencia legal, de modo que el hecho de pertenecer a una organización se convierte en el criterio utilizado para deducir la legalidad de los miembros del sindicato que cuentan con empleo en determinado momento. Es bastante evidente que si la membresía sindical es considerada como el estándar de la condición legal, muchos trabajadores mexicanos ilegales son contados como empleados legales.

Algunos trabajadores mexicanos que inicialmente entraron a los Estados Unidos ilegalmente han llegado a actuar y a desenvolverse tan bien como ciudadanos, que en sus localidades serían contados como

\footnotetext{
"Stoddard, Ellwyn, "A conceptual Analysis of the 'Alen Invasion'Institutionalized Support of Illegal Mexican Aliens in the U.S." International Migration Review. Ne 10, 1976. pp. 166-170 and Sloan, John W. and Jonathan P. Est. "Comunity Integration and Policies among Elites in Two Border Cities: Las Dos Laredes". Journal of Inter-American Studies and World Affairs. Ne 18. And, Sloan, John W. and Jonathan P. West. "The Role of Informal Policy-Making in U.S. Mexico Border Cities". Social Science Quarterly. No 58.
} 
trabajadores legales. Dado que han vivido en una granja en la frontera, han eriado ahí a sus hijos; han desarrollado un alto grado de destreza en el uso de maquinaria agrícola, y cuentan con un grado de habilidad en el dominio de la lengua inglesa que es mejor que el de muchos ciudadanos. Son contados como trabajadores mexicanos legales, aun cuando en la misma familia algunos de los niños hayan nacido antes de la entrada ilegal de sus padres a los Estados Unidos y otros nacieran después. Esto crea una gran tensión dentro de la familia, que está saliendo a relucir como consecuencia de la caída de la nueva legislación Simpson-Mazzoli. Aparentemente, el Servicio de Inmigración de los Estados Unidos ha demorado la acción formal en contra de tales familias que esperan los resultados de las nuevas leyes de inmigración. Pero las siguientes declaraciones de periódicos ilustran el dilema.

Durante ocho años, los Estados Unidos han sido el hogar de Gabriel Licón Navarrete, de 48 de edad. Algunos de sus parientes son ciudadanos estadounidenses. Sus hijos asisten a escuelas públicas en El Paso. El acaba de obtener el mejor trabajo de su vida, laborando en una fábrica de EI Paso por \$ 3.35 dólares la hora. Pero Navarrete y su esposa María son extranjeros ilegales provenientes de México. La mitad de sus seis hijos son ciudadanos estadounidenses, la otra mitad son mexicanos. El 5 de febrero los Navarrete regresarán a México como extraños. Un juez de inmigración ha dictado que deben abandonar los Estados Unidos o ser deportados formalmente.

Se estima que de 3,000 a 5,000 familias más en el área de El Paso se podrían enfrentar a las mismas consecuencias según instrucciones recientes del servicio de Inmigración y Naturalización de los Estados Unidos. El porqué esas órdenes han sido emitidas está en debate ". . . Si la SimpsonMazzoli se hubiera autorizado, estas familias hubieran sido de las primeras en obtener la amnistía". Familias como la de los Navarrete han sido registradas por el SIN y supervisadas regularmente. Ahora, los miembros de las familias que son deportados por un juez de inmigración deben de desarraigarse, de cortar raíces, separarse de sus parientes o de la familia entera y ser enviados a México, perdiendo años de residencia. Esa residencia frecuentemente es requerida por el gobierno federal para que el extranjero legal o ilegal logre ciertos tipos de visas o de naturalización. ". . . Ellos (los del SIN) ya no quieren que esta familia permanezca aquí, durante siete años completos estuvieron esperando para ser los siguientes en turno (al demandar visas para residencia permanente para extranjeros)", decía el Jefe de la Conferencia Católica Estadounidense para Migración y Servicios para Refugiados, quienes ayudan a extranjeros mexicanos ilegales a fin de reunir los requisitos jurídicos para obtener su residencia. ${ }^{9}$

Los estatutos inmigratorios estrictamente jurídicos cortan los sistemas sociales existentes, así como las reglas generales de equidad y justicia. Por eso, ¿es realmente sorprendente que los empleados y patrones

- Heild, Collen. "At Least 3,000 Families May Face Deportation Under New INS Order". El Paso Times. (October 22 1984) 1A/7A. 
reporten a los trabajadores mexicanos como legales, lo sean o no en realidad? En todos los sistemas sociales, cuando las reglas formales o jurídicas se convierten en destructivas para el mantenimiento ordenado y la supervivencia de ese sistema, se desarrollan canales y procedimientos informales a través de los cuales la interacción puede ser efectuada.

Aparentemente, las deformaciones jurídicas que regulan el trabajo legal mexicano son tan incongruentes que llegan a negar sus propios objetivos: mantener un sistema de control ordenado. En este vacío funcionan los trabajadores ilegales mexicanos, ayudados por instituciones norteamericanas y auspiciados por los patrones y los consumidores. De este modo, es difícil decir qué porcentaje de nuestra fuerza de trabajo en el suroeste está compuesta por ilegales frente a los inmigrantes mexicanos legales. Si tal situación es cierta, solamente se pueden obtener aproximaciones generales para obtener el número de trabajadores legales. Y si tal es el caso, la única manera objetiva para estudiar el impacto de los trabajadores mexicanos legales en la economía fronteriza de los Estados Unidos, es la de combinar el mercado de trabajo legal e ilegal y luego separarlos, teniendo en cuenta la tendencia de las instituciones fronterizas de legitimar a los trabajadores ilegales y reportarlos como trabajadores legales.

\section{DISTORCIONES PERCEPTIVAS QUE AFECTAN LA CONDICION LEGAL/ILEGAL DE LOS TRABAJADORES MEXICANOS}

Steiner ${ }^{10}$ sugirió que aunque Los Angeles era la capital de La Raza, para los mexicoamericanos y para los emigrantes mexicanos recién llegados, la región de la frontera era su "cordón umbilical". Esta región está asociada más estrechamente con los mexicanos en Estados Unidos que ninguna otra. Un autor señala:

La mezcla de las costumbres americana y mexicana en esta área, ha dado como resultado una cultura tradicionalmente asociada con el suroeste de los Estados Unidos. La arquitectura, el arte, la literatura y la música de esta región dependen bastante de esta fusión cultural y de las instituciones bilingües y hiculturales que ahí se han desarrollado, y las cuales han sido un campo de entrenamiento para autores mexicoamericanos, así como para educadores y lideres políticos y sociales. Desafortunadamente, esta región fronteriza ha sido también un recurso para la perpetuación de estereotipos étnicos, especialmente los negativos. Con la inmigración constante y la influencia mutua internacional, las diferencias básicas sociales, culturales y jurídicas entre nacionales mexicanos y mexicoamericanos con frecuencia se tornan confusas, especialmente debido a los elementos de prejuicio hacia los mexicoamericanos, que están ya fuertemente establecidos dentro de las estructuras sociales y tradiciones de la región. ${ }^{11}$

10 Steiner, Stan. La Raza: The Mexican Americans. New York: Harper and Row. 1970. p. 141.

"Stoddard, Ellwyn. Mexican Americans. New York, Random House. 1973. p. 30. 
Cuando nos retiramos del asfixiante ambiente ideológico de las politicas jurídicas hacia el nivel mundano de la interacción humana, encontramos que las refinadas distinciones jurídicas formuladas en las leyes de inmigración o de trabajo, que a cada minuto son analizadas en audiencias de cortes de apelación, desaparecen en la realidad del mundo tal y como son percibidas por aquéllos que deben vivir y sobrevivir en cllas. Las instituciones feudales europeas fueron cuidadosamente trasplantadas al nuevo mundo por los conquistadores españoles, y fueron eternizadas por las élites españolas de California, Texas y el territorio de Nuevo México. En la medida en que los angloamericanos empezaron a dominar las instituciones político-jurídicas y económicas de la región, ellos suplantaron a las primeras élites españolas en tanto que retuvieron el papel servil del peón mexicano. $\Lambda$ lo largo del siglo XIX y a principios del XX, este trato continuó sin declinar hasta después de la Segunda Guerra Mundial, cuando la combatibidad étnica dio por resultado la alteración de algunas leyes sobre derechos civiles, el establecimiento de programas de igualdad de oportunidad económica y la determinación de cuotas de las minorías para que a éstas se les diera la oportunidad de ascender en la escala social; así como el énfasis en materia de educación para el progreso de los grupos minoritarios, que en el suroeste incluía a alumnos de habla hispana. Tal y como se pronosticó, esta concentración de apoyo atrajo la atención del público, la cual no toda fue favorable. En la medida en que las oportunidades de educación, de empleo y el apoyo hasado en la necesidad de asistencia se convirtieron en un debate acelerado entre los pagadores de impuestos y los líderes tradicionales, las doctrinas de trato imparcial y repartición justa entraron en conflicto directo. ${ }^{12}$ El incremento de la asistencia social para las familias más pobres de la región, de las cuales un número desproporcionado eran mexicoamericanos ${ }^{13}$ provocó una crítica creciente, a la vez que las reglas de acción acordadas en 1973 por la OPEP (Organización de Países Exportadores de Petróleo) forzaron a la cconomía de Estados Unidos a entrar en recesión. Fuerte fuc el clamor en contra de aquéllos que estaban como sanguijuelas del sistema, y los mexicoamericanos se convirtieron en el chivo expiatorio disponible. Este patrón de conducta, de usar como chivo expiatorio a los extranjeros o a las minorías no populares, es un fenómeno que se repite con frecuencia, incluyendo el comentario de las acciones romanas en contra de los primeros cristianos.

Ellos culpaban a los cristianos de ser la causa de cualquier desastre del estado, o de cualquier desgracia de la gente. Si el Tíber alcanza la pared, si el Nilo no llega a los campos; si el cielo no se mueve o si la tierra

${ }^{12}$ Ryan, William. "The Euality Dilemma". In: Judson R. Landis. Sociology: Concepts and Characteristics. Belmont, Wadsworth Publishing Co. 1981. pp. 155-160.

${ }^{13}$ Stoddard, Ellwyn. Patterns of Poverty along the U.S.-Mexico Border. EI Paso Organization of U.S. Border Cities, University of Texas at El Paso, Center for Inter-American Studies. 1978. 
lo hace; si hay escasez, o si hay una plaga, el clamor es uno: "Los cristianos a los leones". ${ }^{14}$ Por eso cuando el nuevo comisionado de el SIN fue nombrado, el anterior en ese puesto General Chapman, declaró el 22 de octubre de 1974 la siguiente advertencia - la población para ser el chivo expiatorio estaba lista y disponible-. El proclamó:

Estamos siendo invadidos por extranjeros ilegales. Están entrando por millones y sin regulación jurídica, sin dinero y sin fuerza, es poco lo que podemos hacer para detener el flujo. Estamos observando solamente el principio del problema. Cerca del $90 \%$ de los ilegales que aprehendemos vienen de México.

Aunque el número total de inmigrantes mexicanos no se ha incrementado repentinamente a través de los años, esta súbita advertencia creó entre la generalidad de los americanos mucha hostilidad. Cada mexicoamericano - ciudadano, trabajador, estudiante o empleado- se convirtió en "ilegal". Los criterios para obtener la ciudadanía legal no se usaron más. Todas las gentes de origen mexicano, con la excepción de prósperos hombres de negocios, políticos o profesionales, fueron clasificados como parte de la horda invasora de ilegales prometida por el general Chapman en su proclama para que se aumentara el presupuesto del SIN. Muchos de los esfuerzos en materia de trato humanitario de las décadas anteriores se desvanecieron en la medida en que la histeria y los ataques súbitos de los medios de comunicación masiva desplazaron a la tolerancia étnica. No sólo hay mexicanos aquí entre nosotros en donde no son queridos, - dice el planteamiento- pero ciertamente, ellos han profanado nuestra frontera nacional a su paso. Deben ser tratados con rapidez y eficiencia. Esta situación sentó las bases para el famoso incidente de la "cortina de la tortilla" en octubre de 1978, cuando una nueva cerea de alambre para ser instalada por el SIN entre El Paso-Ciudad Juárez y Tijuana-San Isidro, se reportó que era capaz de "cortar un dedo a cualquier extranjero que tratara de escalarla". Esto incitó una situación explosiva entre México y Estados Unidos, y últimamente se ha convertido en un incidente internacional en su máxima expresión. ${ }^{15}$

La principal culpa por la reciente recesión de los Estados Unidos ha sido exitosamente transferida a los extranjeros ilegales en nuestro medio, quienes han obtenido los trabajos y provocado los despidos y el desempleo en Estados Unidos, según dice el planteamiento. Sin embargo, los trabajos han sido perdidos en los centros de producción técnicoindustriales y los extranjeros ilegales todavía están altamente concentra-

\footnotetext{
14 Allport, Gordon W. The Nature of Prejudice. Garden City: Boubleday and Co. (Anchor edition). 1958. p. 235.

is Stoddard, Ellwyn, y otros. El Paso-Ciudad Juarez Relations and the 'Tortilla Courtain': A study of Local Adaptation to Federal Border Policies. El Paso: El Paso Council on the Arts and Humanities. University of Texas at El Paso. 1979.
} 
dos en los sectores de servicios y trabajos agrícolas. Pero con la esperanza de un retorno no doloroso a nuestro anterior período de boom económico, la iniciativa de ley Simpson-Mazzoli fue diseñada para corregir el problema de la inmigración ilegal, principalmente proveniente de México y de sus vecinos del sur. Si bien es cierto que más del 90\% de todos los ilegales mexicanos vienen a los Estados Unidos en busca de trabajo, éstos tienen un impacto mínimo en el desplazamiento de los trabajadores americanos tradicionales en el noroeste. Y su presencia, ya sean aprehendidos o no, ${ }^{16}$ ata a la mayoría de mexicoamericanos e inmigrantes legales mexicanos a la clasificación de "espaldas mojadas"17 y su connotación peyorativa. Si en verdad la conducta humana es controlada por percepciones de la realidad más que por la realidad en sí misma, las consecuencias distorsionadas que suscita acerca de la migración mexicana hacia este país (ambas, legal e ilegal), dan como resultado la discriminación, y las dificultades para esta minoría. La ficción jurídica de separar a los mexicanos "legales" de los mexicanos "ilegales", es completamente nulificada por la forma en que los americanos perciben y tratan a la "gente de piel morena del sur de la frontera".

En suma, ambos, los inmigrantes mexicanos legales y los trabajadores ilegales, se encuentran tan estrechamente integrados dentro de la cconomía fronteriza de Estados Unidos, que cualquier intento hecho para investigar minuciosamente a las gentes hispanoparlantes en el suroeste, a fin de determinar algún teenicismo legal, tendría desvastadoras consecuencias para el suroeste, mismas que serían sentidas en toda la economía norteamericana. Para México, una repentina oleada de repatriación sumergiría la economía de ese país, ya de por sí proble. mática, y sería una carga excesiva para sus limitados servicios públicos. Una perspectiva legal para el "problema mexicano" es casi todo el problema. Continuar con este acertijo ideológico de buscar una solución legal accesible al problema de inmigración mexicana, es demostrar ignorancia respecto a cómo funciona la frontera. Las redes informales fronterizas, institucionalizadas durante décadas, han sido para las gentes de la frontera la única alternativa funcional a fin de mantener sus lazos y culturas fronterizas frente a los esfuerzos legales tendientes a dividir artificialmente la línea de la frontera político-jurídica.

${ }^{16}$ Un gran número de estudios cientificos acerca de los extranjeros ilegales mexicanos aprehendidos y no aprehendidos son citados en Stoddar, Ellwyn. Mexican Migration and Ilegal Immigration. Borderlands Sourcebook. Richard I. Nostrand and Jonathan P. West. (eds.) Norman University of Oklahoma Press. 1983. Cap. 38. También en Cross, Harry E. and James A. Sandos. The Impact of Indocumented Workers the United States: A Critical. Assessment. Washington, D.C. Battelle Human Affairs Research Center. 1979. Se encuentra una excelente bibliografía para examinar este tema.

${ }^{17}$ Bustamante, Jorge A. "The 'wetback' as Deviant: An Application of Labeling Theory" American Journal of Sociology. Ne 77 January, 1972. pp. 706-718. 
Si las políticas de inmigración para el futuro pretenden ser aproximaciones realistas del problema de la migración y del control de la frontera, deben primero entender las realidades funcionales de ésta y formular una política binacional basada en la existencia de los patrones de control informal. Remitirse a las categorías simplistas de legalilegal, es operar dentro de los aislados marcos de trabajo del siglo XIX. El mundo es hoy. Las zonas fronterizas de los Estados Unidos tienen un desarrollo histórico y un sistema que les permite mantener su intercambio social y económico con las de México a pesar de, y no debido a, las políticas federales de inmigración existentes. Es este complejo sistema fronterizo, el que los expertos jurídicos en leyes de inmigración no logran entender e insisten en destruir a fin de conservar la ficción legal de una frontera en la cual dos naciones solamente se tocan, sin traspasarse o interactuar simbióticamente. 


\section{BIBLIOGRAFIA}

ALLPART, Gordon W. The Nature of Prejudice. Garden City: Doubleday and Co. (Anchor Edition). 1958.

BUSTAMANTE, Jorge A. "The 'Wetback' as Deviant: An Application of Labeling Theory". American Journal of Sociology, № 77. January. 1972.

CROSS, Harry and James A. Sandos. The Impact of Undocumentated Workers on the United States: A Critical. Assessment. Washington, D.C. Battelle Human Affairs Research Centers. 1979.

GARCIA y Griego, Manuel. "Mexico and the United States: Migration. History and the Idea of Sovereignty". El Paso, University of Texas at El Paso, Center for Inter-American and Border Studies. Border Perspectives. № 7. December. 1983.

HEILD, Colleen "At Least 3,000 Families May Face Deportation Under New INS Order". El Paso Times. October 22, 1984. 1A/7A.

KEELY, Charles B. "Current Status of U.S. Inmigration and Refugee Policy". In: Mary M. Dritz (ed) U.S. Inmigration and Refugee Policy. Lexington, D.C. Heat (Lexington Books). 1983.

RYAN, William "The Euality Dilemma". In: Judson R. Landis. Sociology: Concepts and Characteristics. Belmont: Wadsworth Publishing Co. 1981.

SLOAN, John W. and West, P., Jonathan "Community Integration and Policies among Elites in Two Border Cities: Los Dos Laredos". Journal of Inter-American Studies and World Affairs. № 18. November, 1976.

"The Role of Informal Policy-Making in U.S.-Mexico Border Cities". Social Science Quarterly. № 58. September, 1977.

STEINER, Stan. La Raza: The Mexican Americans. New York, Harper and Row. 1970.

STODDARD, Ellwyn R. Mexican Americans. New York, Random House. 1973.

"Illegal Mexican Labor in the Borderlands: Institutionalized Support of an Unlawfaul Practice". Pacific Sociological Review. № 19. April. 1976.

"A Conceptual Analysis of the 'Alien Invasion': Institutionalized Support of Illegal Mexican Aliens in the U.S." International Migra. tion Review. № 10.1976.

Patterns of Poverty along the U.S. Mexico Border. El Paso, Organization of U.S. Border Cities, University of Texas at El Paso, Center for Inter-American Studies. 1978.

"Chapter 38: Mexican Migration and Illegal Inmigration". In: Ellwyn R. Stoddard, Richard L. Nostrand and Jonathan P. West (eds.). Borderlands Sourcebook. Norman, University of Oklahoma Press. 1983. 
Functional Dimensions of Informal Border Networks. El Paso, University of Texas at El Paso, Center for Inter-American and Border Studies, Border Perspectives № 8. (January). 1984.

STODDARD, Ellwyn R., Oscar J. Martínez and Miguel Angel Martinez Lasso. El Paso-Ciudad Juarez Relations and the 'Tortilla Curtain': A Study of Local Adaptation to Federal Border Policies. El Paso: EI Paso Council on the Arts and Humanities, University of Texas at El Paso. 1979.

TEITELBAUM, M. "Right versus Right: Inmigration and Refugee Policy in the United States". Foreign Affairs. 59 (№ 1). 1980. 